\title{
Influence of preliminary heat treatment on structure and strength of high-strength aluminum alloy subjected to high pressure torsion with various strains
}

\author{
M. V. Markushev ${ }^{\dagger}$ E. V. Avtokratova, Yu. L. Burdastykh, S. V. Krimsky, O. Sh. Sitdikov \\ †mvmark@imsp.ru
}

Institute for Metals Superplasticity Problems of RAS, 39 S. Khalturin St., Ufa, 450001, Russia

\begin{abstract}
Samples with diam. $20 \mathrm{~mm}$ and thickness $1.5 \mathrm{~mm}$ from a high-strength commercial aluminum alloy 1965 with uni- and bimodal size distributions of nanoscale precipitates of aluminides of transition metals, as well as the main strengthening phase, obtained under preliminary heat treatment, were subjected to high-pressure torsion (HPT) under pressure $6 \mathrm{GPa}$ at room temperature. Number of revolutions was varied in the range from 0.5 to 10 . Methods of transmission electron microscopy and X-ray diffraction analyses were used to certify initial structure-phase states of the alloy and also to investigate the kinetics and mechanisms of deformation structuring of its matrix. On the basis of the data on evolution of structure and hardness of the HPT processed samples, the nature and features of the alloy deformation hardening, depending on heterogeneity of initial structure and strain, were studied. Parameters of the alloy static strength under tension at room temperature were estimated. It was shown that the strain-induced formation of the well-developed nanocellular structure in the alloy matrix may be most effective in terms of its enhanced structural strength: at a rather high level of the alloy hardening obtained, its ductility remained also quite high. Activation of fragmentation and continuous dynamic recrystallization with formation of extremely dispersed nanofragmented and/or nanograined structures, resulted in increased brittleness of failure amid even more enhanced alloy strengthening, as well as to sharp decrease in its ductility, and, hence, in loss of its durability. The role of the dispersed phases and strain in formation of the phase-structural state, ensuring the best balance of the alloy mechanical properties, is discussed.
\end{abstract}

Keywords: aluminum alloy, severe plastic deformation, nanostructuring, strength, ductility, precipitates.

\section{Влияние предварительной термообработки на структуру и прочность высокопрочного алюминиевого сплава, подвергнутого кручению до различных степеней под высоким давлением}

\author{
Маркушев М.В. ${ }^{\dagger}$ Автократова Е. В., Бурдастых Ю.Л., Крымский С. В., Ситдиков О.Ш. \\ Институт проблем сверхпластичности металлов РАН, ул. С. Халтурина, 39, Уфа, 450001, Россия
}

Образцы $\varnothing 20 \times 1.5$ мм из высокопрочного промышленного алюминиевого сплава 1965 с одно- и бимодальным распределением по размерам наноразмерных выделений алюминидов переходных металлов и основной упрочняющей фазы, полученным предварительной термообработкой, были подвергнуты кручению под высоким (6 ГПа) давлением (КВД) при комнатной температуре. При этом количество оборотов изменяли в интервале от 0.5 до 10 . Методами просвечивающей электронной микроскопии и рентгеноструктурного анализа были аттестованы исходные структурно-фазовые состояния сплава, а также изучены кинетика и механизмы деформационного структурирования его матрицы. На основе анализа эволюции структуры и изменений твердости сплава изучены природа и особенности механизмов его деформационного упрочнения в зависимости от гетерогенности исходной структуры и степени 
деформации. Оценены параметры статической прочности сплава при растяжении при комнатной температуре. Показано, что формирование развитой наноячеистой структуры матрицы наиболее эффективно, с точки зрения повышения конструкционной прочности сплава: при сравнительно сильном упрочнении его пластичность сохраняется также на довольно высоком уровне. Активизация при ИПД фрагментации и непрерывной динамической рекристаллизации с формированием предельно диспергированных нанофрагментированных и/или нанозеренных структур, на фоне еще большего упрочнения, охрупчивает сплав и приводит к резкому снижению его пластичности и, соответственно, потере конструкционной прочности. Обсуждена роль дисперсных фаз и степени деформации в формировании структурно-фазового состояния, обеспечивающего наилучший комплекс механических свойств.

Ключевые слова: алюминиевый сплав, интенсивная пластическая деформация, наноструктурирование, прочность, пластичность, вторичные фазы.

\section{1. Введение}

Влияние пластической деформации, реализованной кручением под высоким давлением (КВД) при комнатной температуре, на структуру и механические свойства металлических материалов интенсивно изучается уже более полувека. Непосредственно КВД, как и другим методам так называемой интенсивной (большой, мега, severe и т.п.) пластической деформации (ИПД), посвящена не одна сотня исследований и несколько десятков монографий и обзоров (например, [1-4]). Основное внимание такого рода работ, объединенных общим термином «деформационное структурирование», было уделено предельному диспергированию структуры обрабатываемого материала - приданию ему наноструктурного состояния (с размером структурных элементов менее 0.1 мкм) и, соответственно, его предельному упрочнению за счет измельчения кристаллитов и увеличения протяженности и разориентировки их границ. Однако, опыт промышленной реализации полученных результатов, чаще указывает на нецелесообразность такого подхода, вследствие неоправданной потери конструкционной прочности наноструктурированного материала по причине его сильного охрупчивания.

Представляло интерес выяснить, всегда ли наноструктурирование сплавов, реализованное с помощью КВД, приводит к столь негативному результату или все же есть пути, позволяющие его нивелировать. Задача таких исследований сводится к установлению эффективного пути регламентирования структурно-фазового состояния наноструктурированных материалов, с целью определения основных параметров их структуры, отвечающих за формирование благоприятного комплекса свойств и, прежде всего, - за оптимальный баланс параметров прочности и пластичности при монотонном нагружении при комнатной температуре. Так как наиболее остро такая проблема возникает в отношении трудно-деформируемых сложнолегированных сплавов, в частности, высокопрочных термоупрочняемых сплавов на алюминиевой основе, то важно было определить какой тип наноструктуры предпочтительнее с точки зрения обеспечения их конструкционной прочности в изделиях. При этом необходимо было принять во внимание то, что вследствие преимущественно матричного типа строения этих сплавов, основные технологии изготовления изделий из них позволяли бы обеспечить одновременное двухуровневое наноструктурирование (матрицы и частиц вторых фаз) и, тем самым, улучшить комплекс их свойств за счет сочетания (реализации) трех эффектов: деформационного, дисперсионного и структурного упрочнения.

\section{2. Материал и методы исследования}

В качестве материала исследования был взят промышленный слиток высокопрочного алюминиевого сплава 1965 (Al-8.1Zn-2.2Mg-2.3Cu-0.27Sc-0.10Zr - 0.10Mn, вес.\%). Заготовки диаметром 20 и толщиной 1.5 мм, вырезанные из слитка, гомогенизированного при температуре $450 \pm 5^{\circ} \mathrm{C}$ в течение 24 часов, закаливали в воду с температуры $460^{\circ} \mathrm{C}$, и часть из них затем старили при $170^{\circ} \mathrm{C}$ в течение 5 часов. Все заготовки подвергали КВД от 0.5 до 10 оборотов под давлением 6 ГПа при комнатной температуре.

Тонкую структуру исследовали с использованием просвечивающего электронного микроскопа (ПЭМ) JEOL 2000EX на объектах, подготовленных электрополировкой в растворе 250 мл $\mathrm{HNO}_{3}$ и 750 мл $\mathrm{CH}_{3} \mathrm{OH}$. Размеры и плотность вторичных выделений определяли по снимкам не менее 500 частиц, полученным с участков фольг, имевших близкую толщину. При этом погрешность измерений не превышала $4 \%$.

Рентгеноструктурный анализ (РСА) проводили с помощью дифрактометра ДРОН4 при съемке вращающегося образца в интервале углов $2 \theta=20-40^{\circ}$ при $\mathrm{CuK}_{\alpha}$-излучении, с использованием графитового монохроматора на дифрагированном пучке, при напряжении $40 \mathrm{\kappa B}$, силе тока $20 \mathrm{мA}$, времени экспозиции 5 с, шаге съемки 0.1 град. Полученные спектры обрабатывали в программах OUTSET и PHAN. Параметр решетки а рассчитывали как $a=d \cdot \sqrt{H^{2}+K^{2}+L^{2}}$, где $d$ - межплоскостное расстояние плоскости (HKL). Микродеформацию решетки $\left\langle\varepsilon^{1 / 2}\right\rangle$, области когерентного рассеяния (ОКР) и плотность дислокаций $\rho^{\perp}$, оценивали по величине физических уширений линий.

Микротвердость, HV, сплава определяли на середине радиуса крученых образцов на приборе MVDM 8 при нагрузке 50 г и времени нагружения 15 с по результатам не менее 10-ти измерений на точку. Испытания на растяжение не менее 3-х образцов на точку с определением пределов текучести и прочности и относительного удлинения $\left(\sigma_{0.2}, \sigma_{\text {в }}\right.$, и $\delta$, соответственно), 
проводили на машине Instron 1185 при комнатной температуре на образцах с рабочей частью $1 \times 3$ мм. Ось растяжения была касательной к середине радиуса заготовки.

\section{3. Результаты и обсуждение}

\section{1. Структура и фбазовый состав сплава до и после КВД}

В результате закалки и последующего искусственного старения, в образцах из слитка сплава 1965 с исходным размером зерен около 20 мкм были получены структурные состояния с одно- и бимодальным распределением выделений по размерам, соответственно. А именно, в добавление к когерентным выделениям алюминидов $\mathrm{Zr}$ и Sc, имевшим форму дисков диаметром около 20 нм и плотностью $3 \times 10^{3}$ мкм $^{-3}$ и сравнительно равномерно распределенным в закаленной матрице (пересыщенном основными легирующими элементами алюминиевом твердом растворе), при последующем старении формировались компактные фазы типа $\mathrm{MgZn}_{2}$ размером около 5 нм и плотностью $5 \times 10^{4}$ мкм $^{-3}$ (ПЭМ изображения фаз и их особенности подробно рассмотрены в [5-7]). Таким образом, на стадии предварительной термообработки имел место первый этап наноструктурирования сплава: формирование в матрице наноразмерных вторичных фаз, причем различающихся природой, распределением по размерам и плотностью.

Судя по изображениям на Рис. 1, уже на первом обороте КВД в матрице обоих состояний сплава формировалась развитая, слаборазориентированная, наноячеистая структура. При дальнейшем кручении тип структуры матрицы предварительно состаренного сплава принципиально не изменялся (Рис. 1c,d), вследствие установления динамического равновесия между скоростью генерации дефектов кристаллической решетки и скоростью их аннигиляции за счет динамического возврата. Такое структурирование алюминиевого твердого раствора было обусловлено развитием более гомогенного на микро- и мезоуровне дислокационного скольжения в результате его делокализации при взаимодействии движущихся дислокаций с выделениями двух типов фаз плотностью порядка $10^{4} \mathrm{Mкм}^{-3}$.

Отличительной же особенностью структуры предзакаленного сплава (Рис. 1a, е) было то, что наряду с ячейками в ней выявлялись деформационные полосы, фрагментировавшие исходные зерна. При этом, судя по картинам микродифракции, границы полос, как и границы ячеек, изначально являлись малоугловыми, и с увеличением степени деформации, могли значительно увеличивали разориентировку [8-12].

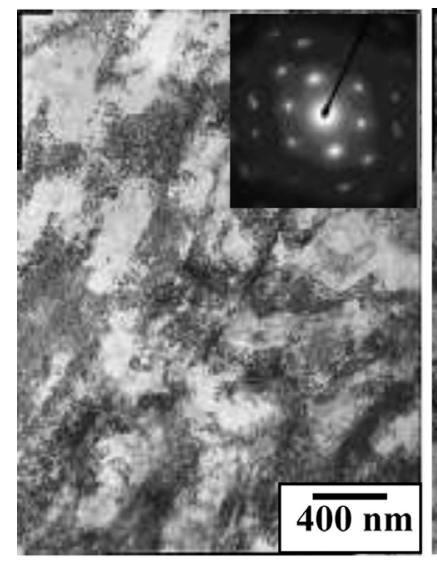

a

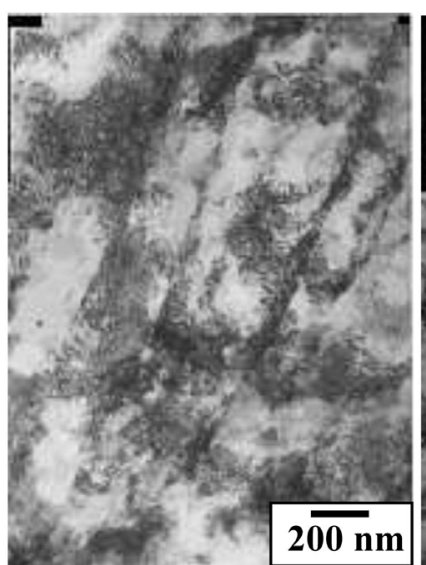

$\mathrm{e}$

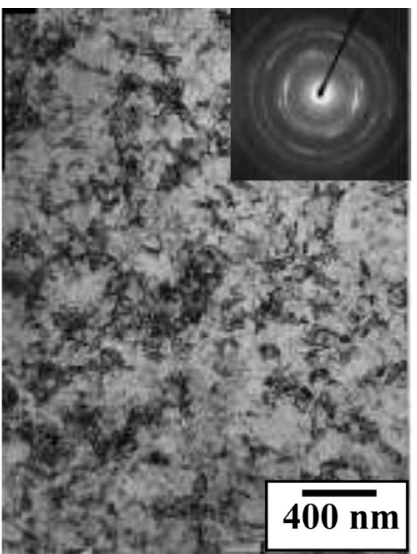

b

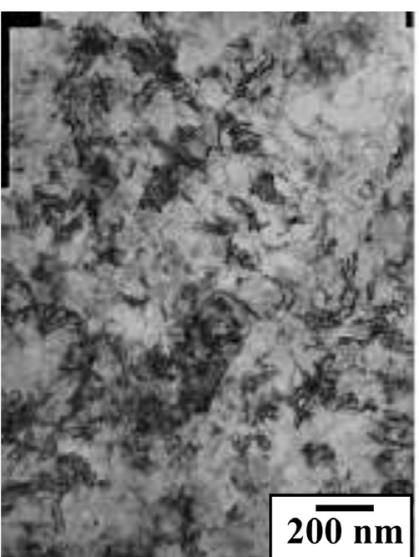

$\mathrm{f}$

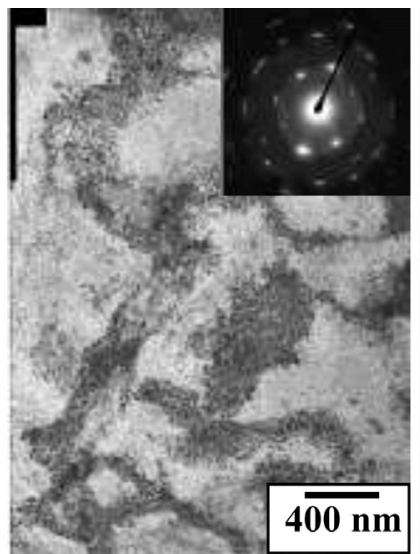

c

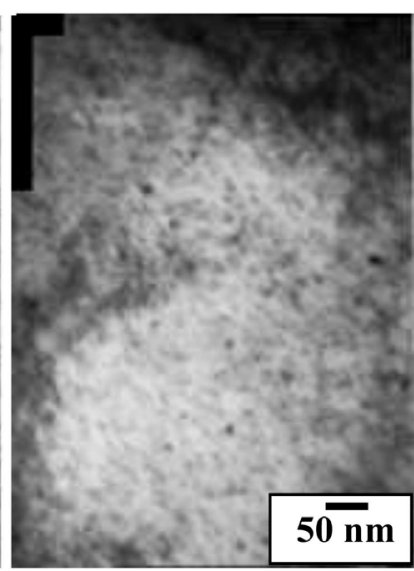

$\mathrm{g}$

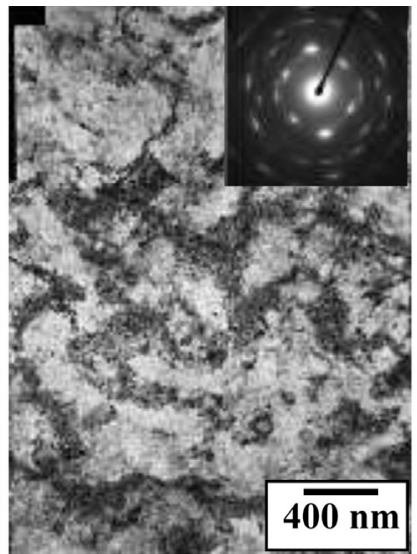

d

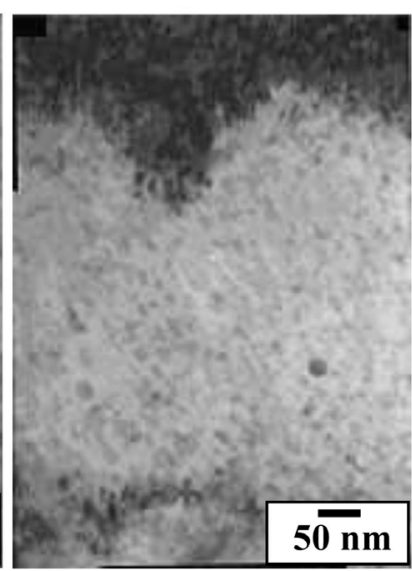

$\mathrm{h}$

Рис. 1. ПЭМ структура закаленного (a, b, e, f) и состаренного (c, d, g, h) сплава после 1 (a, c, e, g) и 5 (b, d, f, h) оборотов КВД. Fig. 1. TEM structure of pre-quenched (a, b, e, f)) and aged (c, d, g, h) alloy after 1 (a, c, e, g) and 5 revolutions (b, d, f, h) under HPT. 
В результате, после 10 оборотов в сплаве фиксировали сравнительно однородную наноструктуру, состоящую преимущественно из фрагментов, окруженных как мало-, так и высокоугловыми границами (Рис. 1b,f) с размером около 80-100 нм. На основании сказанного, можно считать, что именно развитие деформационных полос вносило значительные разориентировки в структуру матрицы и являлось одним из основных факторов, контролирующих формирование новых высокоугловых границ и новых зерен. Таким образом, приблизительно со 2-го оборота в матрице предзакаленного сплава начинался второй этап ее наноструктурирования, приводивший к заметному увеличению доли и разориентировки межкристаллитных границ и трансформации части малоугловых границ в границы общего типа.

Данные РСА (Табл. 1) полностью коррелируют с вышеописанной эволюцией структуры матрицы сплава и свидетельствуют о том, что наиболее значимые изменения ее строения, как то измельчение кристаллитов, искажение кристаллической решетки и накопление дефектов, имеют место на начальном этапе КВД. В дальнейшем структура совершенствуется, причем интенсивнее в предзакаленном сплаве, на что указывают меньшие, чем в состаренном сплаве, микроискажения решетки и плотность дислокаций. Кроме того, по данным РСА параметр решетки в обоих состояниях заметно снижается на начальной стадии КВД, и в дальнейшем изменяется слабо, оставаясь более высоким в закаленном сплаве и сохраняя разницу между состояниями постоянной. Этот результат свидетельствует о незначительном вкладе в изменения этого параметра возможных изменений фазового состава сплава при КВД, причем как с исходно пересыщенной основными легирующими элементами алюминиевой матрицей, так и с уровнем ее легированности, близкой к равновесной, после закалки и старения, соответственно.

В пользу последнего свидетельствовал также ПЭМ контраст от выделений в деформированных заготовках обоих состояний (Рис. 1). Причем в закаленном сплаве, фиксируемый только от алюминидов ПМ, а в состаренном, и от алюминидов, и от выделений фазы $\mathrm{MgZn}_{2}$, выявляемых даже после 10 оборотов КВД. Необходимо отметить, что аналогичную картину наблюдали в [5-7] после старения сплава и по другим режимам. Таким образом, данные ПЭМ и РСА указывали на отсутствие как интенсивного растворения выделений обоих типов при ИПД, так и активного их выделения. Основываясь на этих данных и данных работы [13], можно было полагать, что при деформировании даже предзакаленного сплава с пересыщенной $\mathrm{Zn}, \mathrm{Mg}$ и $\mathrm{Cu}$ алюминиевой матрицей не происходило значимого динамического фазового старения. Этот факт был чрезвычайно важен для понимания природы структуро- и свойствоформирования сплава при КВД.

Интересным результатом было обнаружение меньшего размера ОКР в состаренном сплаве перед- и на начальных этапах ИПД, причем, при больших значениях микроискажений решетки и плотности дислокаций. При этом разница всех этих параметров для обоих исследованных состояний практически нивелировалась с увеличением числа оборотов. Причина заключалась, видимо, в том, что слаборазориентированные блоки, различавшиеся по размеру в исходных состояниях, трансформировались в близкие по размерам дислокационные ячейки, выступавшие основным структурным элементом строения матрицы сплава на начальных этапах деформации. А дальнейшее уменьшение разницы в размерах ОКР, вероятно, было следствием уменьшения расстояния между границами деформационных полос в предзакаленном сплаве, и формирования в нем субзерен (фрагментов), сопоставимых по размеру с ячейками в состаренном сплаве.

\section{2. Механическое поведение сплава}

Судя по Рис. 2, сплав после закалки демонстрировал твердость около $120 \mathrm{HV}$, тогда как последующее дисперсионное твердение упрочняло его почти до 160-170 HV (т. е. на 40 - 50 единиц). Еще большую прочность сплав приобретал в результате КВД, причем наиболее сильно он упрочнялся за первые пол-оборота: твердость в обоих состояниях достигала приблизительно одинаковых значений около $200 \mathrm{HV}$. В дальнейшем твердость состаренных образцов практически не изменялась, что означало достижение установившейся стадии деформирования. А твердость предзакаленных образцов, напротив, продолжала расти и после 2-х оборотов достигала

Табл. 1. Данные РСА сплава до и после КВД.

Table 1. X-ray data for the alloy before and after HPT.

\begin{tabular}{|c|c|c|c|c|}
\hline $\begin{array}{c}\text { Обработка } \\
\text { Тreatment }\end{array}$ & $a, \AA$ & ОКР, нм & $<\varepsilon^{1 / 2}>, \%$ & $\begin{array}{c}\rho^{\perp}, 10^{14} \mathrm{M}^{-2} \\
\rho^{\perp}, 10^{14} \mathrm{~m}^{-2}\end{array}$ \\
\hline $\begin{array}{c}\text { Перед КВД } \\
\text { Веfore НРТ }\end{array}$ & $4.053 \pm 0.001^{\star} / 4.049 \pm 0.001$ & $503 \pm 16 / 100 \pm 7$ & $0.032 \pm 0.001 / 0.002 \pm 0.001$ & $0.08 / 0.02$ \\
\hline $\begin{array}{c}\text { КВД 0.5 об. } \\
\text { НРТ 0.5 rev. }\end{array}$ & $4.049 \pm 0.001 / 4.045 \pm 0.001$ & $73 \pm 2 / 53 \pm 2$ & $0.180 \pm 0.006 / 0.239 \pm 0.009$ & $3.0 / 5.4$ \\
\hline $\begin{array}{c}\text { КВД 2 об. } \\
\text { НРТ 2 rev. }\end{array}$ & $4.049 \pm 0.001 / 4.045 \pm 0.001$ & $61 \pm 2 / 52 \pm 2$ & $0.173 \pm 0.006 / 0.227 \pm 0.009$ & $3.4 / 5.3$ \\
\hline $\begin{array}{c}\text { КВД 10 об. } \\
\text { НРТ 10 rev. }\end{array}$ & $4.048 \pm 0.001 / 4.046 \pm 0.001$ & $57 \pm 2 / 49 \pm 2$ & $0.210 \pm 0.006 / 0.212 \pm 0.004$ & $4.5 / 5.2$ \\
\hline
\end{tabular}

*закаленный / состаренный

* pre-quenched / aged 
порядка $230 \mathrm{HV}$ с последующей ее стабилизацией на этом уровне.

Основываясь на сделанных выше заключениях, можно утверждать, что описанные изменения твердости в большей степени характеризуют стадийность и кинетику наноструктурирования матрицы сплава в процессе КВД, а не изменения его фазового состава, параметров строения вторых фаз или легированности алюминиевого твердого раствора. Об этом же свидетельствует и хорошая корреляция уровня твердости с представленными выше и описанными в $[7,14]$, изменениями структуры других $\mathrm{Al}$ сплавов в зависимости от степени ИПД. Как и в этих работах, упрочнение сплава 1965 в обоих состояниях на начальном этапе КВД было вызвано дислокационным и субструктурным упрочнением вследствие формирования близкой по природе дислокационной / ячеистой структуры, которая в дальнейшем слабо изменялась в состаренном состоянии и не приводила к значительным изменениям его твердости. В закаленном же сплаве, в условиях развитой ИПД, протекала фрагментация исходных зерен и непрерывная динамическая рекристаллизация. При этом его твердость росла как за счет появления новых границ, так и увеличения их разориентировок. То есть, КВД состаренного сплава приводило к деформационному упрочнению за счет повышения плотности дислокаций и формирования наноячеистой структуры, которое по величине было соразмерно эффекту искусственного старения сплава. А формирование нанофрагментированной структуры и реализация зернограничного и дислокационного упрочнения при КВД предзакаленного сплава, при сохранении твердорастворного упрочнения матрицы, дополнительно придавали ему еще большую твердость, причем примерно на ту же величину - 40 - 50 HV.

Из Рис. 2 и Табл. 2 следует, что в отличие от твердости, прочностные характеристики при растяжении предзакаленного сплава были выше уже после 0.5 оборота, несмотря на то, что состаренный сплав был дисперсно упрочнен перед КВД почти на максимальную величину. При этом пластичность в обоих состояниях была высокой для столь сильноупрочненного сплава. После одного оборота прочность обоих состояний уменьшилась, причем предзакаленного состояния сильнее, что практически нивелировало их разницу. После 2-х оборотов, прочностные характеристики сплава вновь возрастали, причем в состоянии после закалки они достигали аномально высоких значений для данного сплава, превысив 900 МПа, и увеличив разницу в пределе текучести до 215 МПа. Однако, столь сильное упрочнение сплава снизило его пластичность более чем в три раза. На фоне дальнейшего незначительного роста параметров прочности и резкого снижения удлинений состаренного сплава, прочность закаленного сплава после 5 оборотов снова снижалась. Причем предзакаленый сплав охрупчивался столь сильно, что разрушался с нулевыми удлинениями в упруго-пластической области, т.е. при значительно меньших напряжениях, соответствующих всего лишь пределу прочности Т1 стандартно термообработанного сплава [15]. Немногим большую пластичность (поряд-

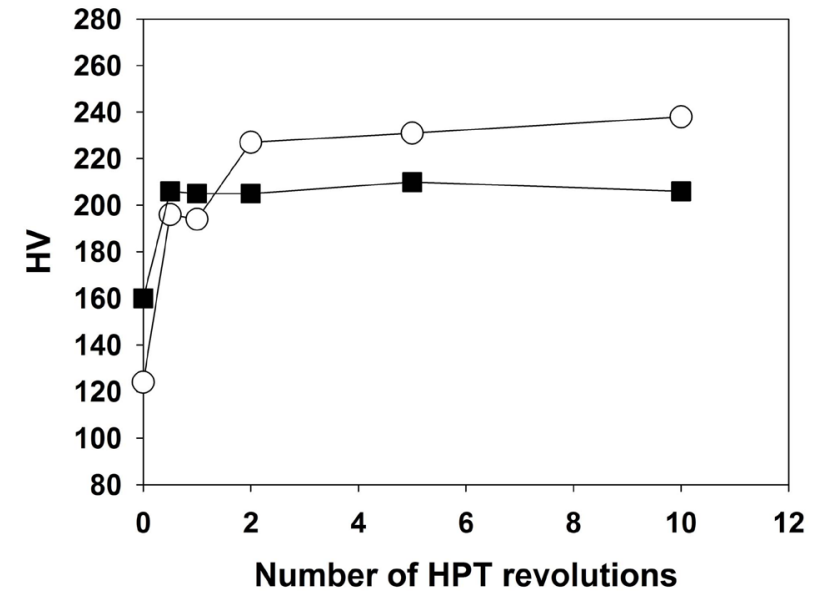

Рис. 2. Зависимость твердости закаленного (о) и состаренного (ש) сплава от количества оборотов при КВД.

Fig. 2. Hardness of pre-quenched ( $($ ) and aged ( $\mathbf{a})$ alloy vs number of HPT revolutions.

Табл. 2. Свойства при растяжении при комнатной температуре. Table 2. Room temperature tensile strength.

\begin{tabular}{|c|c|c|c|}
\hline $\begin{array}{l}\text { КВД, об. } \\
\text { НРТ, rev. }\end{array}$ & $\begin{array}{l}\sigma_{0.2}, \mathrm{M \Pi а} \\
\mathrm{YS}, \mathrm{MPa}\end{array}$ & $\begin{array}{c}\sigma_{\mathrm{s}}, \mathrm{M} \mathrm{a} \\
\mathrm{UTS}, \mathrm{MPa}\end{array}$ & $\begin{array}{l}\delta, \% \\
\mathrm{El}, \%\end{array}$ \\
\hline 0.5 & $730 / 685^{\star}$ & $780 / 720$ & $8.5 / 9.0$ \\
\hline 1 & $590 / 610$ & $640 / 650$ & $9.0 / 7.0$ \\
\hline 2 & $900 / 685$ & $960 / 720$ & $2.5 / 6.5$ \\
\hline 5 & $-/ 770$ & $850 / 810$ & $-/ 1.0$ \\
\hline 10 & $-/ 750$ & $640 / 800$ & $-/ 1.0$ \\
\hline \multicolumn{4}{|c|}{ Свойства листа сплав 1965 T1 [15] } \\
\hline \multicolumn{4}{|c|}{ Properties of the 1965 T6 sheet [15] } \\
\hline & 625 & 650 & 16.3 \\
\hline
\end{tabular}

* закаленный/состаренный

${ }^{*}$ pre-quenched/aged

ка 1\%) фиксировали в состаренном сплаве, когда предел его прочности приблизился к 800 МПа. При этом надо принять во внимание тот факт, что структура его матрицы не претерпевала явных признаков фрагментации с преобразованием ячеистой структуры в нанофрагментированную, как при КВД предзакаленного сплава.

\section{4. Выводы}

1. Результаты работы свидетельствуют о важности контроля исходной гетерогенности структуры при термомеханической обработке высокопрочных алюминиевых сплавов типа 1965, проводимой с целью наноструктурирования их матрицы с использованием методов ИПД, в частности, кручения под высоким давлением при комнатной температуре.

2. Показано, что наноразмерные выделения алюминидов переходных металлов и основной упрочняющей фазы играют в сплавах роль не только упрочняющего агента, но и выступают одним из ключевых факторов, контролирующих наноструктурирование их матрицы в обработках с ИПД, приводящих к формированию как развитых дислокационных (ячеистых), так и фрагментированных (зеренно-субзеренных) структур. 
3. На примере сплава 1965 установлено, что формирование в теромоупрочняемых сплавах развитой наноячеистой структуры более эффективно, чем нанофрагментированной с точки зрения обеспечения регламентировано высокого их упрочнения при сохранении довольно высокой пластичности. С большой вероятностью можно утверждать, что достигнутый уникальный баланс прочности и пластичности будет обеспечивать и высокие параметры механического поведения сплава, коррелирующие с ним, такие как ударная вязкость, сопротивление усталости и другие. Напротив, развитие при ИПД фрагментации и непрерывной динамической рекристаллизации с формированием предельно диспергированных нанофрагментированных и нанозеренных структур, приводит к резкому снижению и прочности, и пластичности сплава при растяжении, и соответственно, к потере потенциала его конструкционной прочности.

Благодарности/Acknowledgements. Работа выполнена при поддержке гранта РНФ №16-19-10152 П и в рамках работ ИПСМ по основным заданиям РАН (AAAA-A19-119021390107-8). / The study was performed under the support of RSF Frant № 16-19-10152P and in the frame of basic researches of RAS (AAAA-A19-119021390107-8).

\section{Литература}

1. R.Z. Valiev, R.K. Islamgaliev, I.V. Alexandrov. Progr. Mater. Sci. 45, 103 (2000). Crossref

2. T. G. Langdon. Acta Mater. 61, 7035 (2013). Crossref

3. Y. Estrin, A. Vinogradov. Acta Mater. 61, 782 (2013), Crossref

4. R. R. Mulyukov, R. M. Imayev, A. A. Nazarov, M. F. Imayev, V.M. Imayev. Superplasticity of Ultrafine Grained Alloys: Experiment, Theory, Technologies. Nauka,
Moscow (2014) 284 p. (in Russian) [P. Р. Мулюков, Р. М. Имаев, А. А. Назаров, М. Ф. Имаев, В. М. Имаев. Сверхпластичность ультрамелкозернистых сплавов: эксперимент, теория, технологии. Наука, Москва (2014) 284 c.]

5. M.V. Markushev, E.V. Avtokratova, S.V. Krymskiy, O. Sh. Sitdikov. J. Alloys Compd. 743, 773 (2018). $\underline{\text { Crossref }}$

6. M.V. Markushev, Y.L. Burdastykh, S.V. Krymskiy, O.S. Sitdikov. Lett. Mater. 7 (2), 101 (2017). $\underline{\text { Crossref }}$

7. M. V. Markushev, E. V. Avtokratova, O. Sh. Sitdikov. Lett. Mater. 7 (4), 459 (2017). $\underline{\text { Crossref }}$

8. P. J. Apps, M. Berta, P. B. Prangnell. Acta Mater. 53, 499 (2005). Crossref

9. N.A. Koneva, E. V. Kozlov. Tambov State Univer. Bull. 8 (4), 514 (2003). (in Russian) [Н. А. Конева, Э. В. Козлов. Вестн. Тамб, Гос. у-та. 8 (4), 514 (2003).]

10. O.S. Sitdikov. Lett. Mater. 5 (1), 74 (2015). (in Russian) [О. Ситдиков. Письма о материалах. 5 (1), 74 (2015).] Crossref

11. T. Sakai, A. Belyakov, H. Miura. Metall. Mat. Trans. 39, 2206 (2008). Crossref

12. P. J. Hurley, F. J. Humphreys. Acta Mater. 51, 1087 (2003). Crossref

13. S. V. Krymskiy, D. K. Nikiforova, M. Yu. Murashkin, M. V. Markushev. Prosp. Mater. 12, 387 (2011). (in Russian) [C.В. Крымский, Д.К. Никифорова, М. Ю. Мурашкин, М.В. Маркушев. Перспективные материалы. 12, 387 (2011).]

14. O. Sitdikov, S. Krymskiy, M. Markushev, E. Avtokratova, T. Sakai. Rev. Adv. Mater. Sci. 31, 62 (2012).

15. V.I. Elagin, L. B. Ber, T.D. Rostova, E. I. Shvechkov, O. G. Ukolova. Techn. of Light Alloys. 2, 20 (2013). (in Russian) [В.И. Елагин, Л.Б. Бер, Т.Д. Ростова, Е.И. Швечков, О.Г. Уколова. Технология легких сплавов. 2, 20 (2013).] 